\title{
Journal of The American Society for Mass Spectrometry
}

\section{Supporting Information}

\section{Online Monitoring the Key Intermediates and Volatile Compounds Evolved from Green Tea Roasting by Synchrotron Radiation Photoionization Mass Spectrometry}

Keke Qi, ${ }^{a}$ Minggao Xu, ${ }^{\text {a }}$ Hao Y in, ${ }^{\mathrm{b}}$ Liutian $\mathrm{Wu},{ }^{\mathrm{a}}$ Yonghua Hu,${ }^{\mathrm{c}}$ Jiuzhong Yang, ${ }^{\mathrm{a}}$ Chengyuan Liu, *,a and Yang Pan*,a

a National Synchrotron Radiation Laboratory, University of Science and Technology of China, Hefei 230029, China, E-mail: 1cy2012@ustc.edu.cn; pangyang@ustc.edu.cn.net, Tel: +86-551-63601986.

${ }^{b}$ National Laboratory for Physical Sciences at Microscale, University of Science and Technology of China, Hefei, Anhui 230026, P.R. China.

${ }^{c}$ Research and Development Centre, China Tobacco Anhui Industrial Co., Ltd., Hefei, 230088, China.

*Prof. Yang Pan, Email: panyang@ustc.edu.cn

*Dr. ChengyuanLiu, Email:1cy2012@ustc.edu.cn 

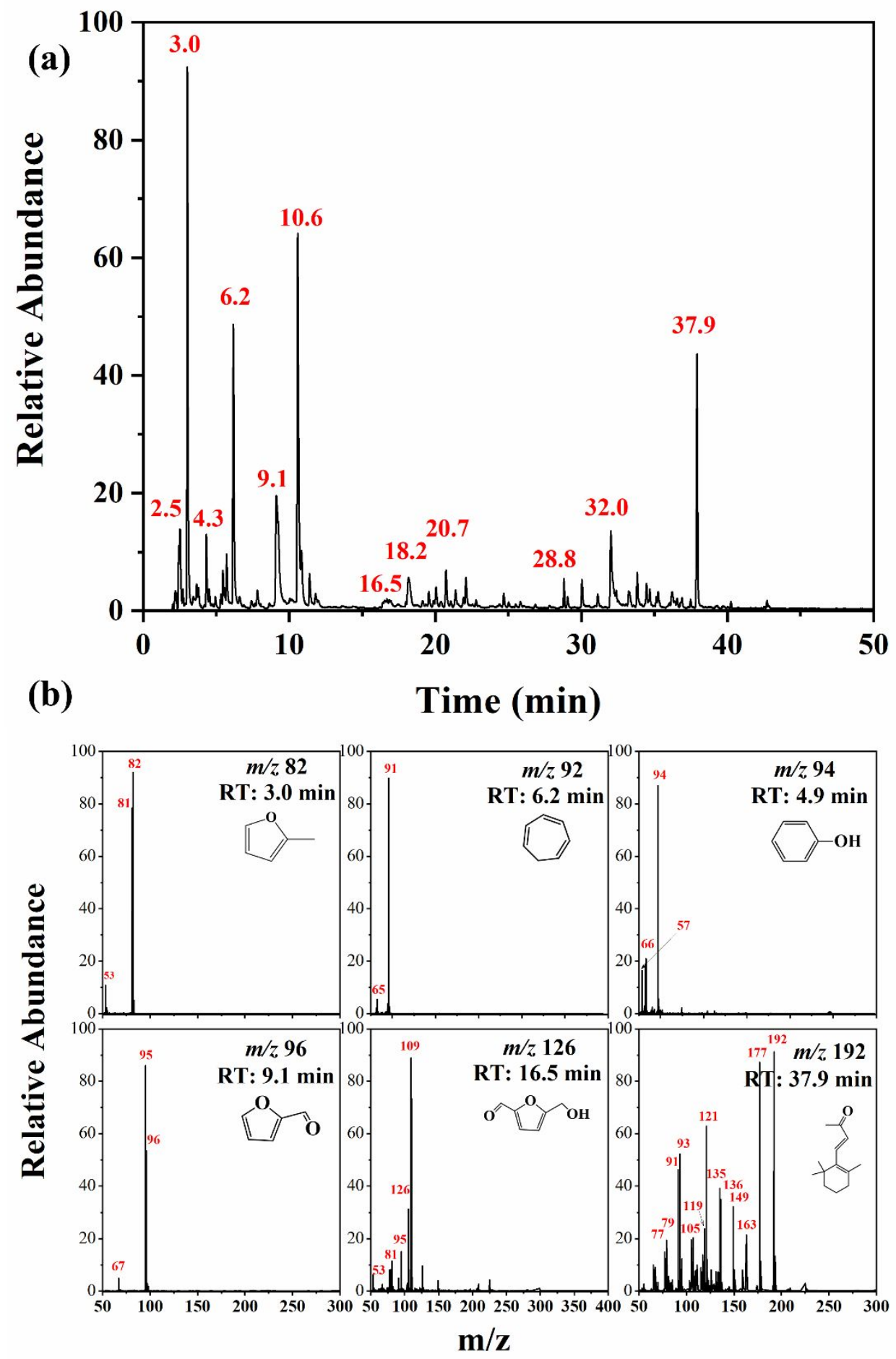

Figure S1. (a) The total ion chromatogram of the gaseous species during the roasting of green tea by headspace GC-MS. (b) The mass spectra of some representative compounds selected from the chromatography at the corresponding retention time. 


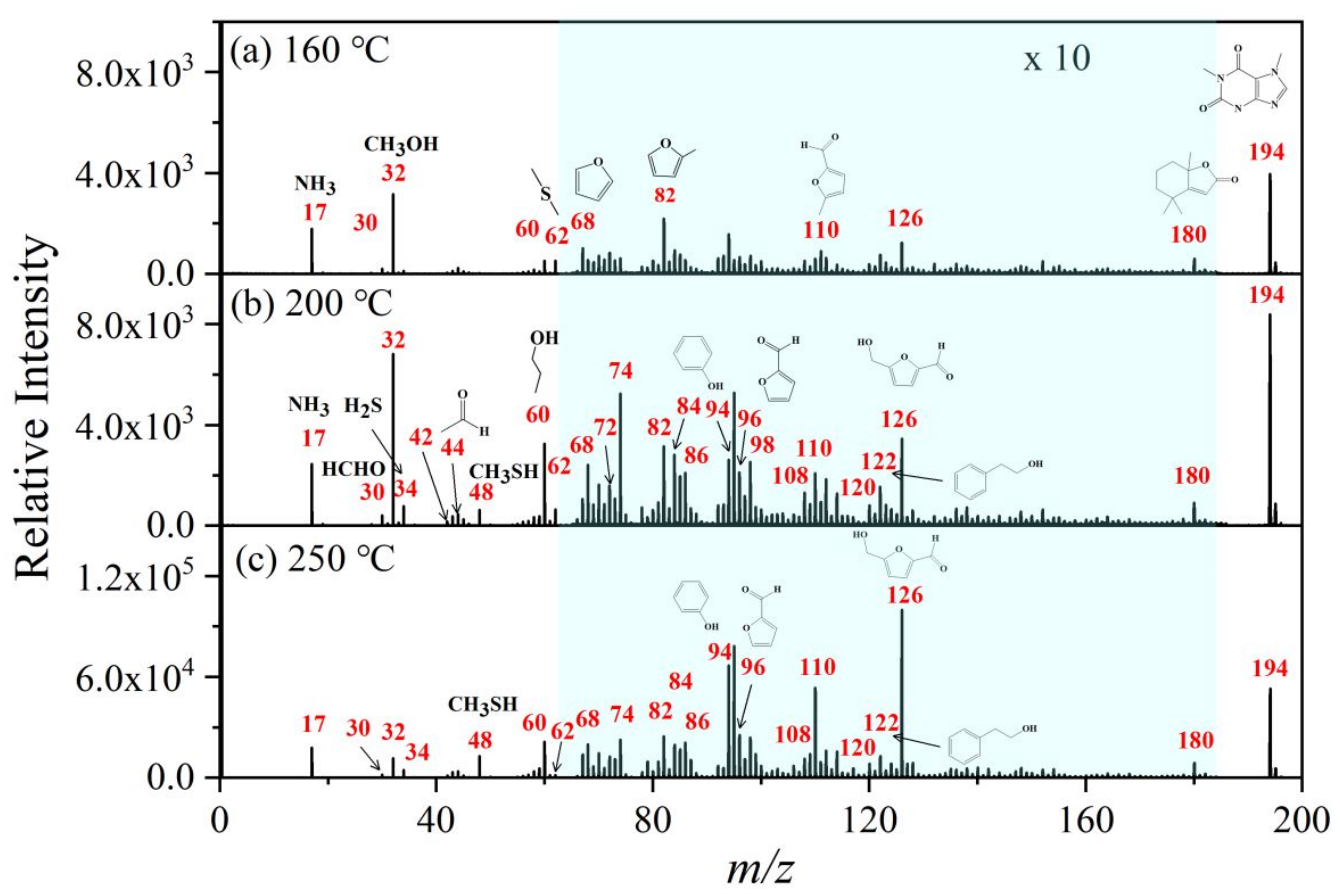

Figure S2. The accumulated photoionization mass spectra of the evolved gas during the roasting of fresh green tea with $11 \mathrm{eV}$ photon energy at different roasting temperature: (a) $160{ }^{\circ} \mathrm{C}$, (b) $200{ }^{\circ} \mathrm{C}$ and (c) 250 ${ }^{\circ} \mathrm{C}$. 


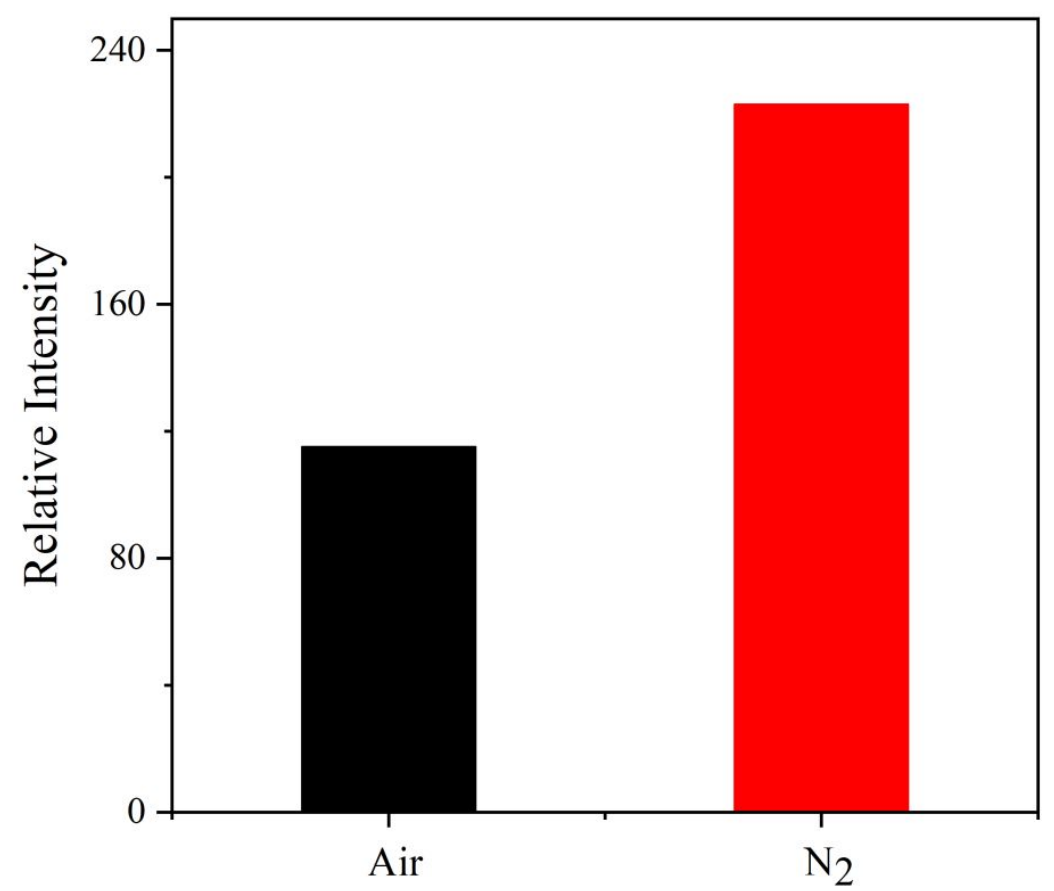

Figure S3. The ion intensities of 5-HMF at $m / z 126$ at the atmosphere of air and $\mathrm{N}_{2}$ under $11 \mathrm{eV}$ photon energy and $200{ }^{\circ} \mathrm{C}$ were compared when $20 \mathrm{mg} 5$-HMF was added in the sample boat for analysis. 


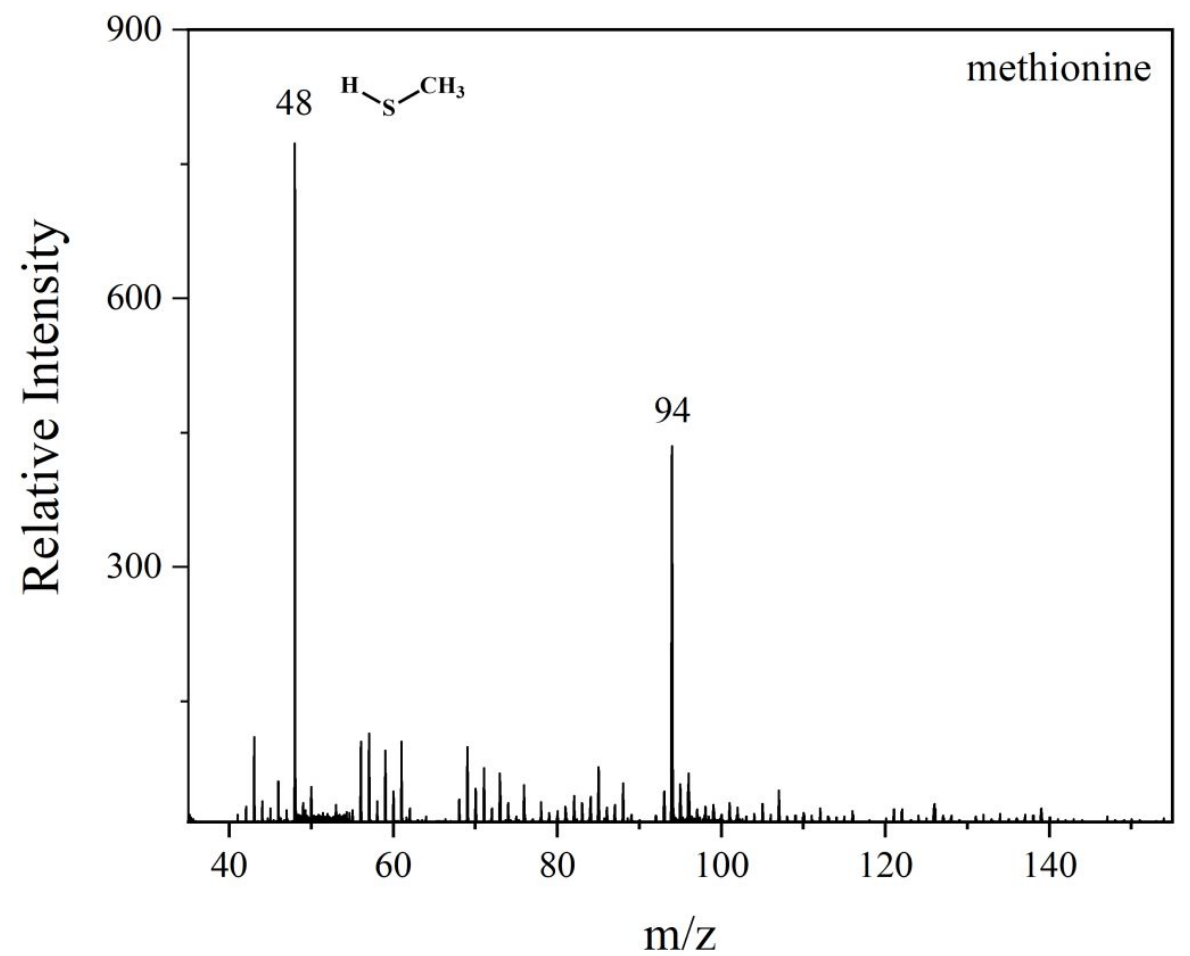

Figure S4. The accumulated photoionization mass spectra during the pyrolysis of methionine at $200{ }^{\circ} \mathrm{C}$ and $11 \mathrm{eV}$ photon energy.
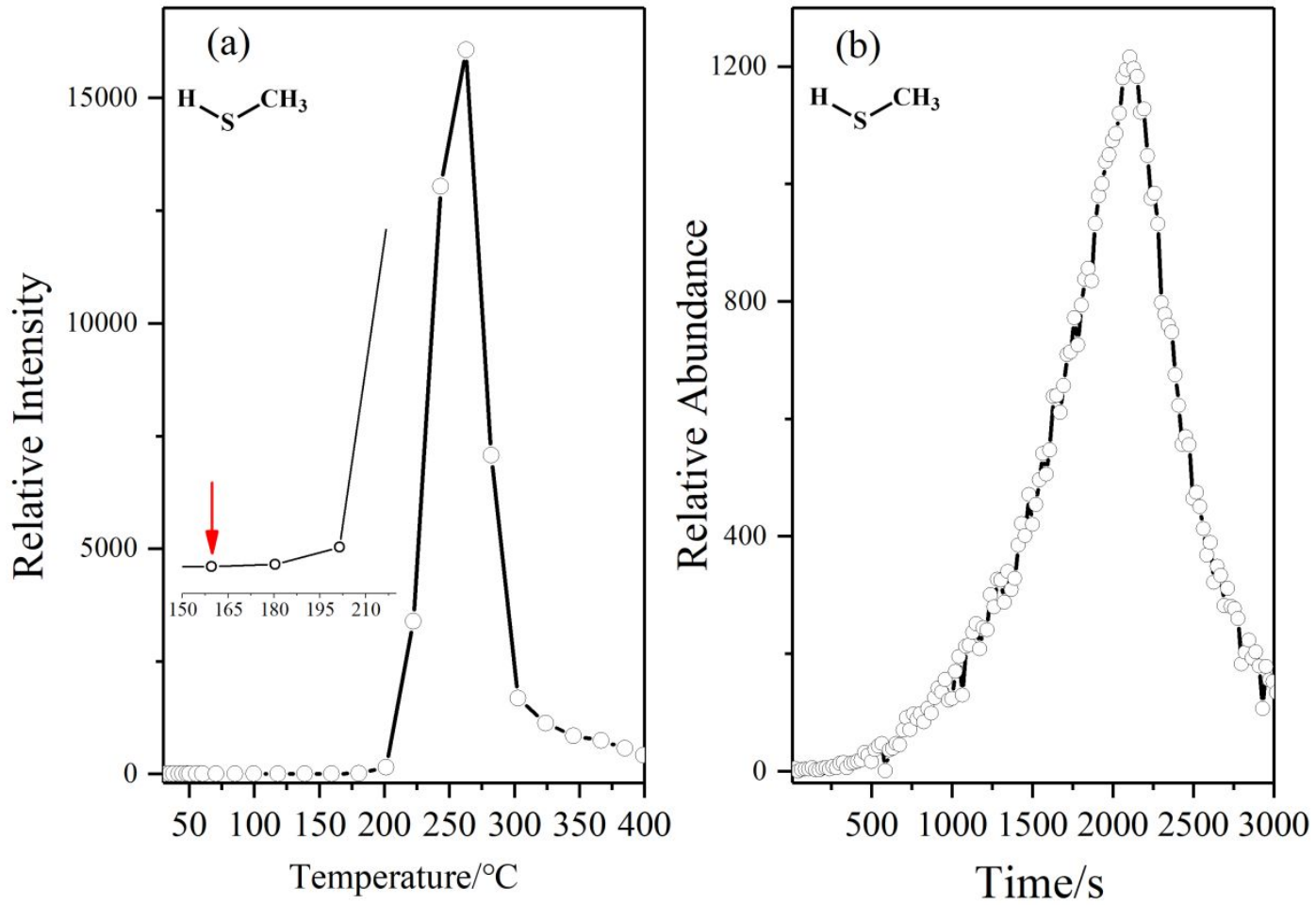

Figure S5. When air was used as carrier gas and $11 \mathrm{eV}$ photon energy was applied, (a) temperatureresolved and (b) time-resolved profiles of product, methanethiol $(\mathrm{m} / \mathrm{z} 48)$ were obtained during the roasting of methionine. For time-resolved experiment, the temperature of furnace was set at $200{ }^{\circ} \mathrm{C}$. 

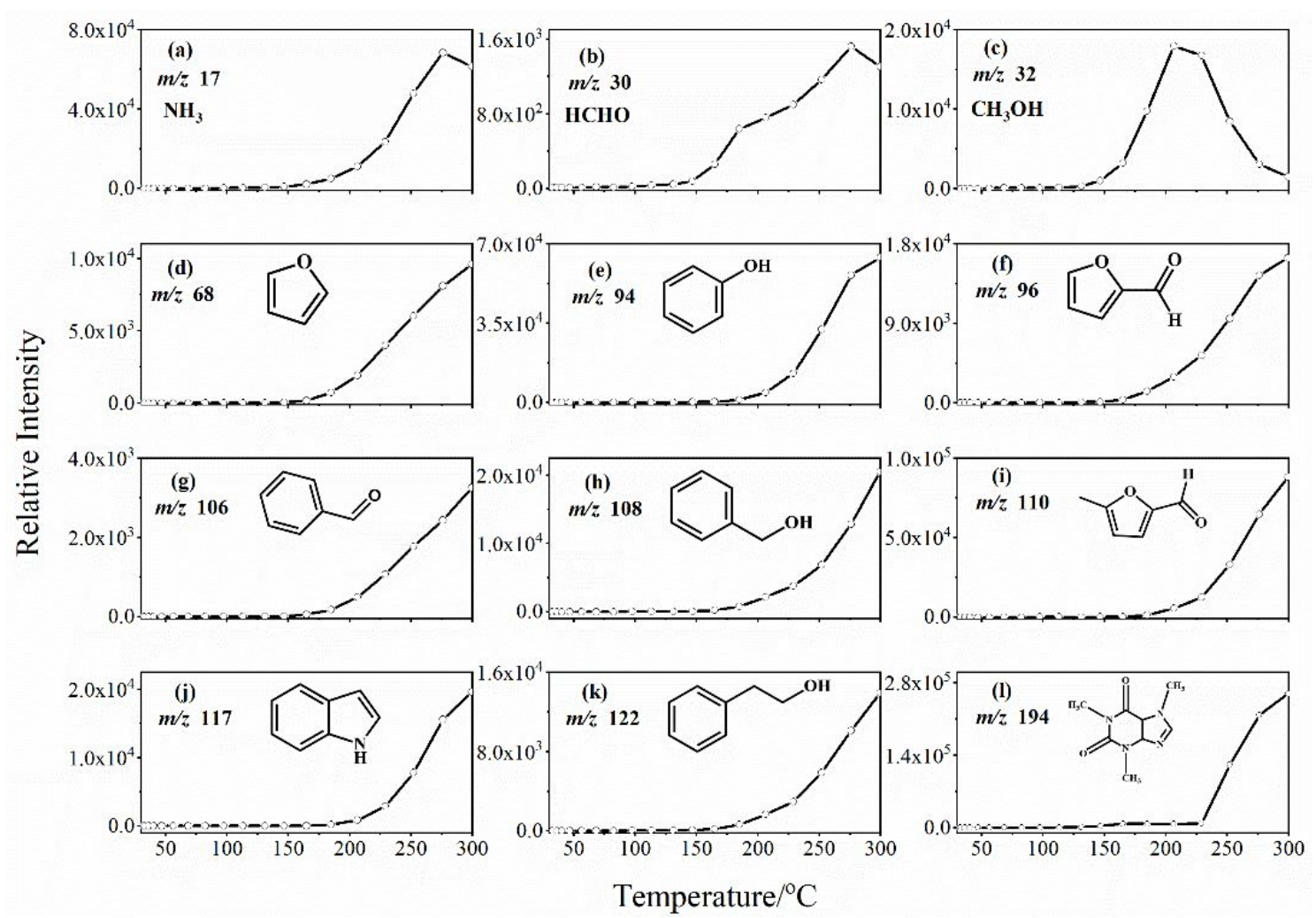

Figure S6. The temperature-resolved profiles of some other volatile compounds during the roasting of green tea were presented. Air was used as carrier gas and $11 \mathrm{eV}$ photon energy was selected for ionization. 